\title{
FUTURE CONTRIBUTIONS TO $\phi_{s}$ MEASUREMENTS*
}

\author{
VARVARA BATOZSKAYA \\ on behalf of the LHCb Collaboration \\ National Centre for Nuclear Research (NCBJ), Warszawa, Poland \\ varvara.batozskaya@cern.ch
}

(Received May 10, 2018)

The most recent results on the $\mathrm{CP}$-violating phase $\phi_{s}$ measurements in the $B_{s}^{0}-\bar{B}_{s}^{0}$ system obtained by the LHCb Collaboration with Run 1 data are presented. Thanks to the precise prediction of the $\phi_{s}$ value in the frame of the Standard Model, it represents an excellent probe to search for new physics. Current results are compatible with the Standard Model predictions, and their precision will be increased with larger data sets. Further improvement is expected from the inclusion of results obtained using decay modes with smaller branching fractions.

DOI:10.5506/APhysPolB.49.1015

\section{Introduction}

The phase $\phi_{s}$ can be related to the angle $\beta_{s}$ of the unitary CKM triangle of the $B_{s}^{0}$ meson system analogous to $\beta$ angle in $B^{0}$ meson decay [1]. The interference between the mixing and direct decay of $B_{s}^{0}$ mesons to CP eigenstates via $b \rightarrow c \bar{c} s$ transitions allows to measure the CP-violating phase, $\phi_{s}$ :

$$
\phi_{s}=\phi_{\mathrm{M}}-2 \phi_{\mathrm{D}}=-2 \beta_{s}+\Delta \phi_{s}^{\text {Peng }}+\delta_{s}^{\mathrm{NP}},
$$

where $\phi_{\mathrm{M}}$ and $\phi_{\mathrm{D}}$ are the mixing and direct phases, respectively. The other components in the $\phi_{s}$ determination are higher order "pengiun" diagrams from non-perturbative hadronic effects (Fig. 1) and new physics (NP) contributions that could be difficult to distinguish from "penguins". These components start to play an important role when reaching high precision of the $\phi_{s}$ measurement [2].

Including only the dominant "tree-level" contributions (Fig. 1), the phase $\phi_{s}$ within the Standard Model (SM) is predicted to be $-2 \beta_{s}$, where $\beta_{s}=$ $\arg \left(-V_{t s} V_{t b}^{*} / V_{c s} V_{c b}^{*}\right)[3]$. An indirect determination of $2 \beta_{s}=37.6_{-0.7}^{+0.8} \mathrm{mrad}$ is obtained using a global fit to experimental data [4].

* Presented at the Cracow Epiphany Conference on Advances in Heavy Flavour Physics, Kraków, Poland, January 9-12, 2018. 


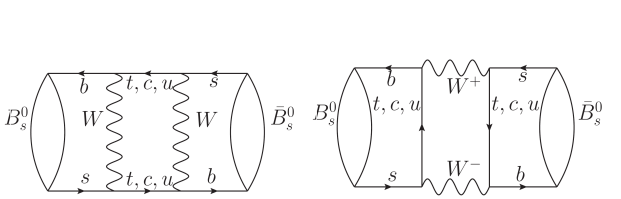

(a) (b)

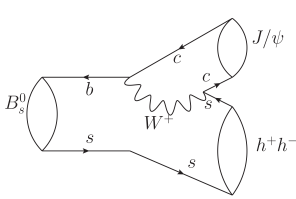

(c)

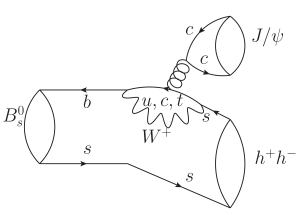

(d)

Fig. 1. Feynman diagrams: (a)-(b) $B_{s}^{0}-\bar{B}_{s}^{0}$ mixing and contributions to the decay $B_{s}^{0} \rightarrow J / \psi h^{+} h^{-}$within the SM, where $h=\pi, K$ : (c) "tree-level", and (d) "penguin" diagrams.

The initial measurements of the phase $\phi_{s}$ have been performed by the Tevatron experiments: CDF [5] and D $\emptyset$ [6] with large uncertainties. Extensive studies have been made by the LHC experiments: ATLAS [7], CMS [8] and LHCb [9] with Run 1 data collected in 2011 and 2012 at a center-ofmass energy of $\sqrt{s}=7 \mathrm{TeV}$ and $8 \mathrm{TeV}$, respectively. At the LHCb [10], the measurement of the CP-violating phase $\phi_{s}$ has independently been performed using $B_{s}^{0} \rightarrow D_{s}^{+} D_{s}^{-}$[11], $B_{s}^{0} \rightarrow J / \psi \phi[9], B_{s}^{0} \rightarrow J / \psi \pi^{+} \pi^{-}$[12], $B_{s}^{0} \rightarrow \psi(2 S) \phi[13]$, and $B_{s}^{0} \rightarrow J / \psi K^{+} K^{-}$with $m\left(K^{+} K^{-}\right)$above the $\phi(1020)$ mass [14] decay modes. The world average value shown in Fig. 2 is consistent with the SM predictions but the combined measurement is still far from the SM precision thus leaving still some room for NP effects. Improvements on the sensitivity of $\phi_{s}$ are expected from the inclusion of Run 2 data collected in 2015-2019 at a center-of-mass energy of $\sqrt{s}=13 \mathrm{TeV}$ that will allow to use the $b \rightarrow c$ and $b \rightarrow s$ processes with very small branching fraction to constrain the $\phi_{s}$ measurement. These proceedings discuss some of those decay modes as a future potential to the determination of the $\mathrm{CP}$-violating phase $\phi_{s}$.

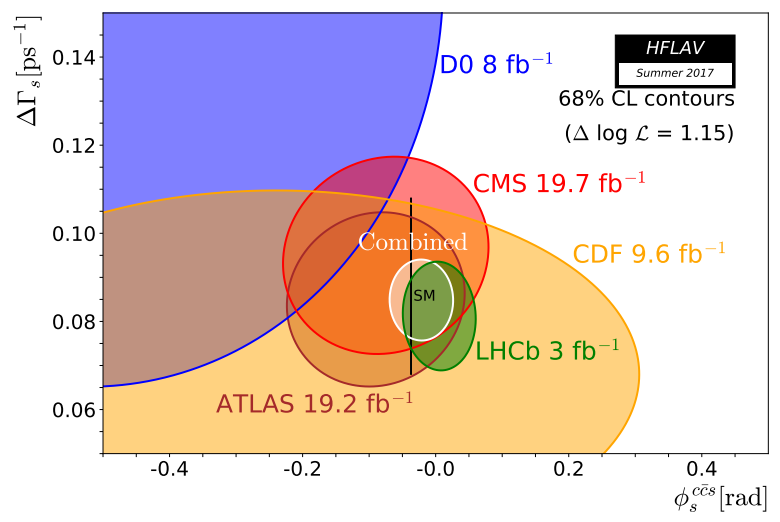

Fig. 2. (Colour on-line) Regions of $68 \%$ confidence level in $\Delta \Gamma_{s}$ and $\phi_{s}$ plane obtained from individual contours of CDF, D $\emptyset$, CMS, ATLAS and LHCb measurements and the combined contour (solid line and shaded area) [15]. The expectation within the SM [4] is shown as a black thin rectangle. 


\section{Observation of the $B_{s}^{0} \rightarrow \eta_{c} \phi$ decay}

For the first time the $\mathrm{LHCb}$ Collaboration has observed the decay $B_{s}^{0} \rightarrow$ $\eta_{c} \phi$, where the $\eta_{c}$ meson is reconstructed in the $p \bar{p}, K^{+} K^{-} \pi^{+} \pi^{-}, \pi^{+} \pi^{-} \pi^{+} \pi^{-}$ and $K^{+} K^{-} K^{+} K^{-}$decay modes and the $\phi(1020)$ in the $K^{+} K^{-}$decay mode using Run 1 data [16]. The $B_{s}^{0} \rightarrow J / \psi \phi$ decay with the same final states is used as a normalization channel. The interference between the $\eta_{c}$ and purely nonresonant contributions is taken into account using an amplitude model to simultaneously fit the four hadrons and $p \bar{p}$ mass distributions (Fig. 3). The branching fractions are measured to be $\mathcal{B}\left(B_{s}^{0} \rightarrow \eta_{c} \phi\right)=[5.01 \pm 0.53 \pm 0.27 \pm$ $0.63] \times 10^{-4}$ and $\mathcal{B}\left(B_{s}^{0} \rightarrow \eta_{c} \pi^{+} \pi^{-}\right)=[1.76 \pm 0.59 \pm 0.12 \pm 0.29] \times 10^{-4}$. In both cases, the first uncertainty is statistical and the second is systematic. The third uncertainty is due to the limited knowledge of the branching fractions of the normalisation channel. In the future, with significant improvement of the hadronic trigger efficiencies [17], these decay modes may become of interest to add sensitivity to the measurement of $\phi_{s}$.
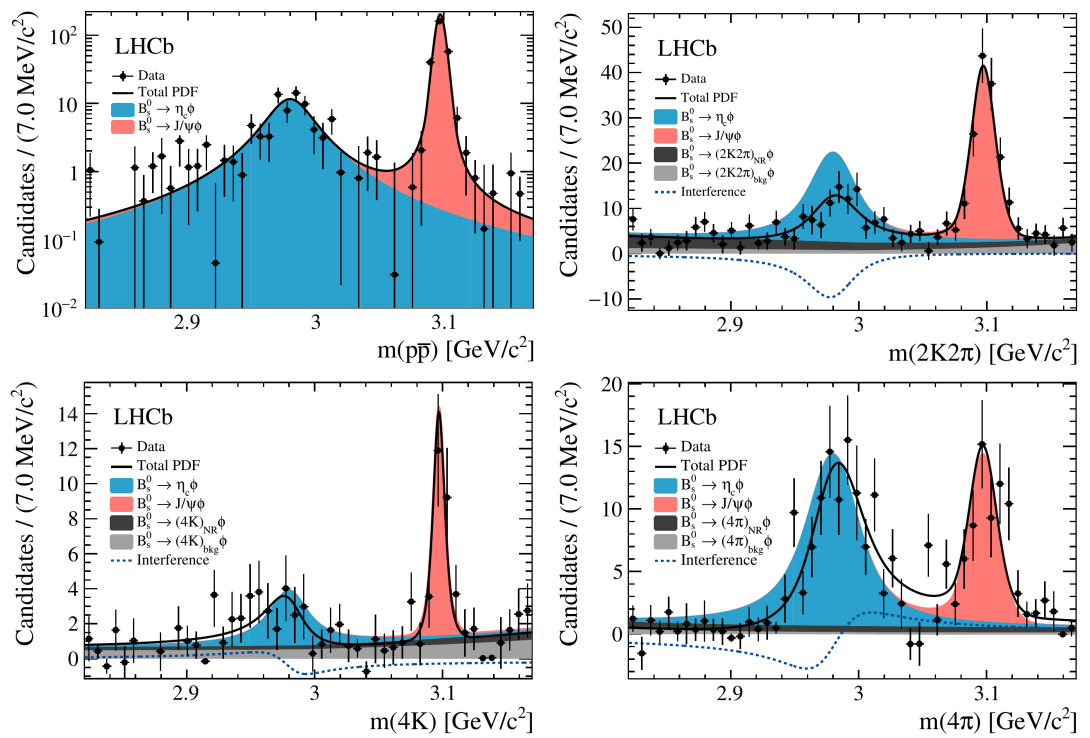

Fig. 3. (Colour on-line) Invariant mass distributions for selected $p \bar{p}, K^{+} K^{-} \pi^{+} \pi^{-}$, $K^{+} K^{-} K^{+} K^{-}$and $\pi^{+} \pi^{-} \pi^{+} \pi^{-}$candidates presented as the left peak (blue area). The normalization channel shows as the right peak (red area). The nonresonant contributions and background candidates describe by dark and light grey area, respectively.

\section{The $B_{s}^{0} \rightarrow J / \psi \eta$ effective lifetime}

The $B_{s}^{0}$ effective lifetime has been measured by the LHCb Collaboration using CP-even $B_{s}^{0} \rightarrow J / \psi \eta(\rightarrow \gamma \gamma)$ decay mode, with $J / \psi \rightarrow \mu^{+} \mu^{-}$and $\eta \rightarrow$ 
$\gamma \gamma$ using Run 1 data [18]. As $\phi_{s}$ is measured to be small and assuming CP conservation, the effective lifetime corresponds to the lifetime of the light $B_{s}^{0}$ mass eigenstate, $\tau_{L} \propto \Gamma_{L}$. The invariant mass resolution is approximately $48 \mathrm{MeV} / c^{2}$ (Fig. 4) causing the overlap of the $B_{s}^{0}$ signal mode with the $B^{0} \rightarrow J / \psi \eta$ background component. The effective lifetime for $\sim 3000$ signal candidates is measured to be $\tau_{\text {eff }}=1.479 \pm 0.034 \pm 0.011 \mathrm{ps}$. The result is consistent with, and has a similar precision to, the other CP-even lifetime measurements [19, 20].
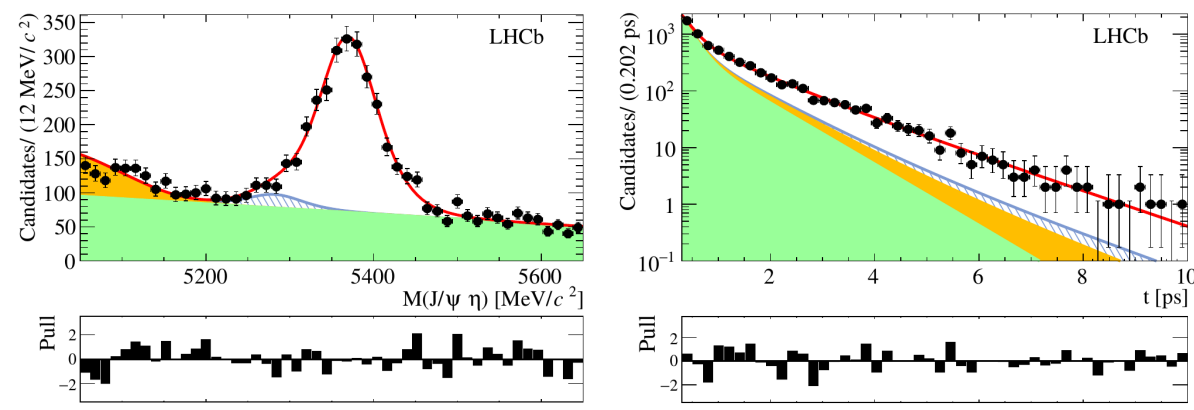

Fig. 4. (Colour on-line) Distributions of $J / \psi \eta$ invariant mass (left) and decay time (right) for selected $B_{s}^{0} \rightarrow J / \psi \eta$ decays. Combinatorial background (light grey/green), background from $B^{0} \rightarrow J / \psi \eta$ decays (hatched/blue) and partially reconstructed background (dark grey/orange) are shown.

\section{Observation of the $B_{s}^{0} \rightarrow \phi \pi^{+} \pi^{-}$decays}

The first observation of the inclusive decay $B_{s}^{0} \rightarrow \phi\left(\rightarrow K^{+} K^{-}\right) \pi^{+} \pi^{-}$ has been performed by the LHCb Collaboration [21]. Figure 5 shows the result of the final fit to the $m\left(K^{+} K^{-} \pi^{+} \pi^{-}\right)$distribution. The $B_{s}^{0}$ yield is found to be around 700 signal candidates. Since the $\pi^{+} \pi^{-}$spectrum includes several resonances, an amplitude analysis to the $\pi^{+} \pi^{-}$mass and decay angle distributions is used to separate out exclusive contributions to the $B_{s}^{0}$ meson decays (Fig. 5). The $B_{s}^{0} \rightarrow \phi \phi$ is used as a normalization channel for both the inclusive and exclusive decays. The decays $B_{s}^{0} \rightarrow$ $\phi f_{0}(980), B_{s}^{0} \rightarrow \phi f_{2}(1270)$ and $B_{s}^{0} \rightarrow \phi \rho^{0}$ are observed with a significance of $8 \sigma, 5 \sigma$ and $4 \sigma$, respectively. The measurement of their branching fractions is $\mathcal{B}\left(B_{s}^{0} \rightarrow \phi f_{0}(980)\right)=\left[1.12 \pm 0.16_{-0.08}^{+0.09} \pm 0.11\right] \times 10^{-6}, \mathcal{B}\left(B_{s}^{0} \rightarrow \phi f_{2}(1270)\right)=$ $\left[0.61 \pm 0.13_{-0.05}^{+0.12} \pm 0.06\right] \times 10^{-6}$ and $\mathcal{B}\left(B_{s}^{0} \rightarrow \phi \rho^{0}\right)=[2.7 \pm 0.7 \pm 0.2 \pm 0.2] \times 10^{-7}$, where the first uncertainty is statistical, the second is systematic, and the third is related to the knowledge of the normalization channel branching fraction. The measurements are consistent with the SM predictions and, in the case of the $B_{s}^{0} \rightarrow \phi \rho^{0}$, they provide a constraint on possible contributions from NP effects [22]. 

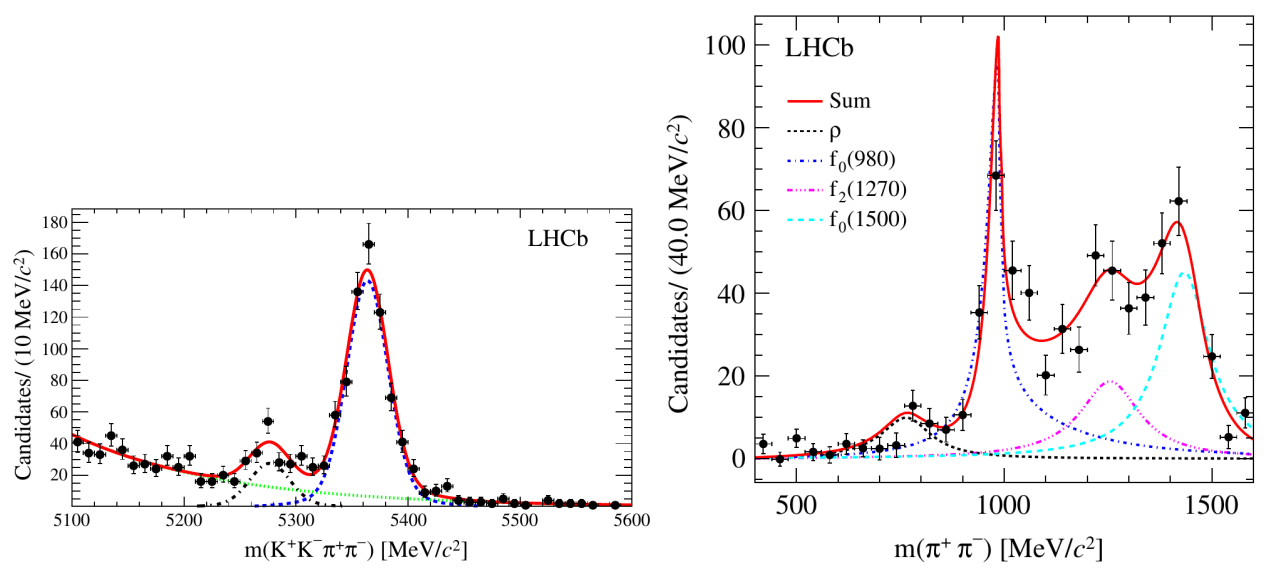

Fig. 5. (Colour on-line) (Left) Distribution of $K^{+} K^{-} \pi^{+} \pi^{-}$invariant mass where the dashed (blue) line is the $B_{s}^{0}$ signal, the dotted (green) line shows the combinatorial background and the dot-dashed (black) line indicates the $B^{0}$ component. (Right) Distributions of $\pi^{+} \pi^{-}$invariant mass with contributing components.

\section{Summary}

The recent measurements of the $\mathrm{CP}$-violating phase $\phi_{s}$ in the $B_{s}^{0}$ meson system, dominated by the LHCb experiment, are compatible with the SM predictions. The addidtional data from Run 2 of the LHC is expected to improve the current precision significantly. New decay modes have been investigated to either measure CP-violating effects or make preparations for such measurements in the future. The statistical sensitivity of the $\phi_{s}$ measurement after the LHCb Phase 1 upgrade in 2021-2029, with an integrated luminosity of $46 \mathrm{fb}^{-1}$, is expected to be $\sim 0.01 \mathrm{rad}$, close to the present the-

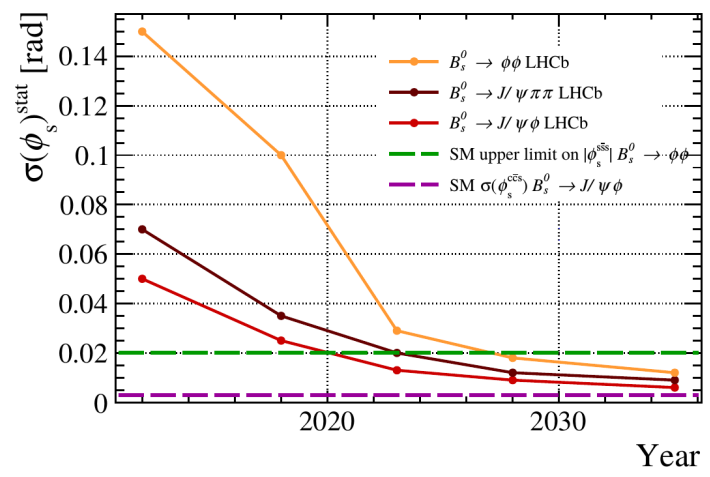

Fig. 6. Projection of how precision on $\phi_{s}$ from LHCb measurements will scale as a function of time for different decay modes. Information taken from Ref. [23]. 
oretical uncertainty (Fig. 6) [24]. As the experimental precision improves, the higher order "penguin" contributions to the $B_{s}^{0}$ decays need to be kept under control and are expected to be no greater than 20 mrad [25, 26].

V.B. is supported by the National Science Centre, Poland (NCN) under the contract UMO-2015/18/M/ST2/00123. The author wishes to thank the organizers of the Epiphany conference for the amazing atmosphere in Kraków and her $\mathrm{LHCb}$ colleagues who helped in the preparation of this paper.

\section{REFERENCES}

[1] N. Cabibbo, Phys. Rev. Lett. 10, 531 (1963).

[2] Z. Ligeti, M. Papucci, G. Perez, Phys. Rev. Lett. 97, 101801 (2006); P. Ball, R. Fleischer, Eur. Phys. J. C 48, 413 (2006); A. Lenz, Phys. Rev. D 76, 065006 (2007); R. Fleischer, eConf C 0610161, 020 (2006); U. Nierste, Int. J. Mod. Phys. A 22, 5986 (2007).

[3] M. Kobayashi, T. Maskawa, Prog. Theor. Phys. 49, 652 (1973).

[4] J. Charles et al., Phys. Rev. D 91, 073007 (2015).

[5] T. Aaltonen et al. [CDF Collaboration], Phys. Rev. Lett. 109, 171802 (2012).

[6] V.M. Abazov et al. [D $\emptyset$ Collaboration], Phys. Rev. D 85, 032006 (2012).

[7] G. Aad et al. [ATLAS Collaboration], J. High Energy Phys. 1608, 147 (2016).

[8] V. Khachatryan et al. [CMS Collaboration], Phys. Lett. B 757, 97 (2016).

[9] R. Aaij et al. [LHCb Collaboration], Phys. Rev. Lett. 114, 041801 (2015).

[10] A.A. Alves, Jr. et al. [LHCb Collaboration], JINST 3, S08005 (2008).

[11] R. Aaij et al. [LHCb Collaboration], Phys. Rev. Lett. 113, 211801 (2014).

[12] R. Aaij et al. [LHCb Collaboration], Phys. Lett. B 736, 186 (2014).

[13] R. Aaij et al. [LHCb Collaboration], Phys. Lett. B 762, 253 (2016).

[14] R. Aaij et al. [LHCb Collaboration], J. High Energy Phys. 1708, 037 (2017).

[15] Y. Amhis et al., arXiv:1612.07233 [hep-ex].

[16] R. Aaij et al. [LHCb Collaboration], J. High Energy Phys. 1707, 021 (2017).

[17] LHCb Collaboration, CERN-LHCC-2014-016, LHCB-TDR-016.

[18] R. Aaij et al. [LHCb Collaboration], Phys. Lett. B 762, 484 (2016).

[19] R. Aaij et al. [LHCb Collaboration], Phys. Rev. Lett. 112, 111802 (2014).

[20] R. Aaij et al. [LHCb Collaboration], Phys. Lett. B 736, 446 (2014).

[21] R. Aaij et al. [LHCb Collaboration], Phys. Rev. D 95, 012006 (2017).

[22] L. Hofer, D. Scherer, L. Vernazza, J. High Energy Phys. 1102, 080 (2011).

[23] LHCb Collaboration, LHCb-PUB-2014-040.

[24] A. Bharucha et al. [LHCb Collaboration], Eur. Phys. J. C 73, 2373 (2013).

[25] R. Aaij et al. [LHCb Collaboration], J. High Energy Phys. 1511, 082 (2015).

[26] R. Aaij et al. [LHCb Collaboration], Phys. Lett. B 742, 38 (2015). 